\title{
Mammographic Features of Hidradenitis Suppurativa
}

\author{
Santo Maimone*, Michelle D. McDonough \\ Department of Radiology, Mayo Clinic Florida, Jacksonville, USA \\ Email: *santomaimoneiv@gmail.com
}

Received September 10, 2012; revised October 14, 2012; accepted October 26, 2012

\begin{abstract}
Hidradenitis suppurativa is a chronic suppurative inflammation of the apocrine sweat glands. Axillary, inframammary, intermammary, and peri-areolar apocrine gland involvement may be visualized mammographically. Characteristic lesions tend to be round or oval, lucent, and smooth-bordered, with or without central densities. While diagnosis made purely via mammography is unlikely, having an awareness of this entity may prove useful in patient care in regards to proper utilization of both imaging and consultative resources.
\end{abstract}

Keywords: Hidradenitis Suppurativa; Mammogram; Breast

\section{Case Report}

A 58-year old woman was referred to our facility seconddary to repeated cyst formation and sinus tract drainage within the right axilla. Further inquiry revealed a longstanding history of recurrent cysts within the bilateral axillary and inguinal regions, as well as reported interventions upon innumerable cysts throughout her body. Despite frequent attempts at incision and drainage in addition to prior treatments with isotretinoin and doxycycline, formation of cysts persisted. She was given the provisional diagnosis of hidradenitis suppurativa over two decades ago, with concomitant bacterial infections overriding the diagnostic consideration of Fox-Fordyce disease.

\section{Discussion}

Hidradenitis suppurativa is a chronic suppurative inflammation of the apocrine sweat glands. Affected apocrine glands tend to occur within the axillary, inguinal, and perineal regions but may also be seen within the inframammary folds, around the areolae, and between the breasts [1,2]. Hidradenitis suppurativa is a purely clinical diagnosis characterized by painful, recurring, inflammatory papules and nodules with acute or chronic evolution that may result in fistula formation and tissue distortion. It occurs in approximately $1 \%$ of the population and is more common in women, with initial onset typically seen between puberty and age 40 [3]. Known associated diseases include inflammatory bowel disease, spondyloarthropathies, as well as SAPHO (synovitis-acne-pustulosis-hyperostosis-osteitis) syndrome. Aggravating fac-

\footnotetext{
*Corresponding author.
}

tors include obesity, skin irritation, shaving, stress, excessive perspiration, heat, and hormonal alterations [4].

Exclusive breast involvement of hidradenitis suppurativa is exceedingly rare, and most occurrences will involve concurrent axillary disease. A distant commentary discussing the mammographic features of hidradenitis suppurativa, written over 20 years ago, described the pathognomonic axillary lesion as a lucent mass with a small central density [5]. Mammographic appearance of hidradenitis suppurativa is nearly impossible to distinguish from that of oil cysts, especially without the circumferential calcifications seen in chronic oil cysts. Lesions are likely to appear round or oval, lucent, and smooth-bordered, with or without a small central density (Figures 1 and 2). In addition to oil cysts, the most
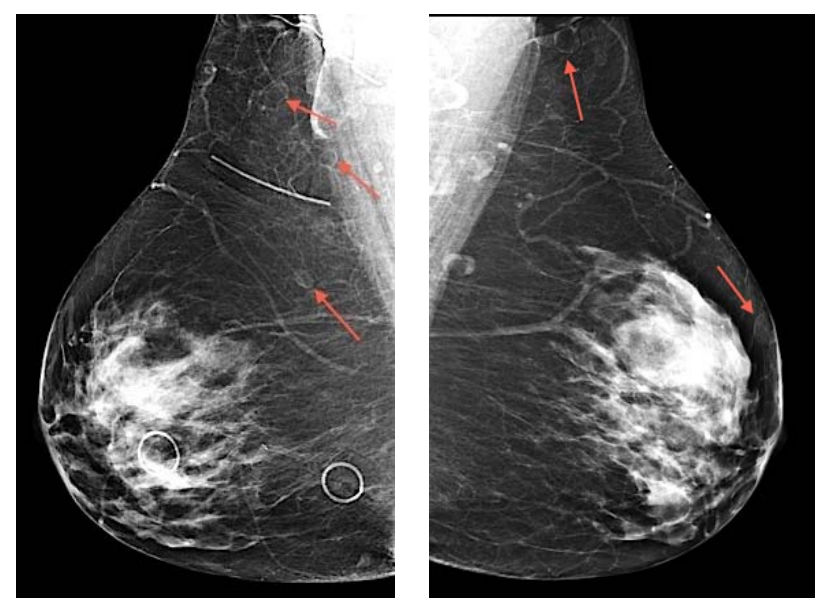

Figure 1. Bilateral mediolateral oblique mammographic images demonstrating numerous oval, lucent, smooth-bordered lesions (arrows) within the axillae and throughout the breast parenchyma. 


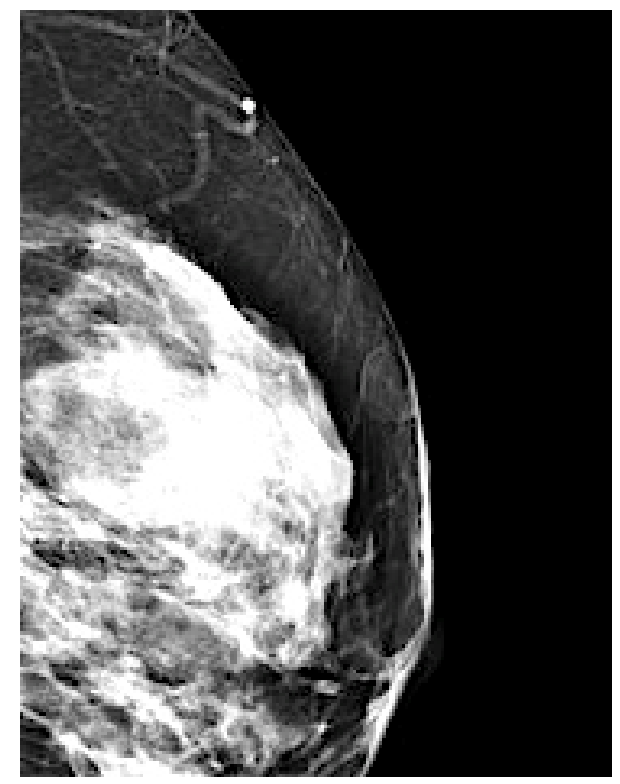

Figure 2. Magnified left MLO mammographic image demonstrating rounded lesion abutting the skin surface of the breast parenchyma.

common differential considerations seen on mammography include lipomas and benign lymph nodes.

Hidradenitis suppurativa can result in significant pain and diminished quality of life. Management is challenging, as the disease is often reluctant to respond to medication and may recur after surgical excision. Following initial consultation with dermatology, our patient was referred for plastic surgery consultation and ulti- mately underwent wide local excision and defect closure of the right axilla.

Solely mammographic diagnosis of hidradenitis suppurativa is unlikely. However, the radiologist can keep this diagnostic consideration in mind, especially in cases of diffuse breast involvement or situations in which clinical history may be unavailable. This may ultimately provide better direction of patient care and appropriate management of both imaging and consultative resources.

\section{REFERENCES}

[1] B. N. Vasconcelos, J. C. Fonseca and D. L. Obadia, "Case for Diagnosis,” Anais Brasileiros de Dermatologia, Vol. 86, No. 3, 2011, pp. 601-602. doi:10.1590/S0365-05962011000300034

[2] A. McMichael, D. Guzman Sanchez and P. Kelly, "Folliculitis and the Follicular Occlusion Tetrad,” 2nd Edition, Mosby Elsevier, St. Louis, 2008, pp. 39, 517-530.

[3] H. S. Smith, J. D. Chao and J. Teitelbaum, "Painful Hidradenitis Suppurativa," The Clinical Journal of Pain, Vol. 26, No. 5, 2010, pp. 435-444. doi:10.1097/AJP.0b013e3181ceb80c

[4] D. L. Whitaker-Worth, V. Carlone, W. S. Susser, N. Phelan and J. M. Grant-Kels, "Dermatologic Diseases of the Breast and Nipple," Journal of the American Academy of Dermatology, Vol. 43, No. 5, 2000, pp. 733-754. doi:10.1067/mjd.2000.109303

[5] L. Kalisher and A. Dembner, "Mammographic Features of Hidradenitis Suppurativa Axillaris,” Canadian Association of Radiologists Journal, Vol. 41, No. 3, 1990, pp. 160-161. 